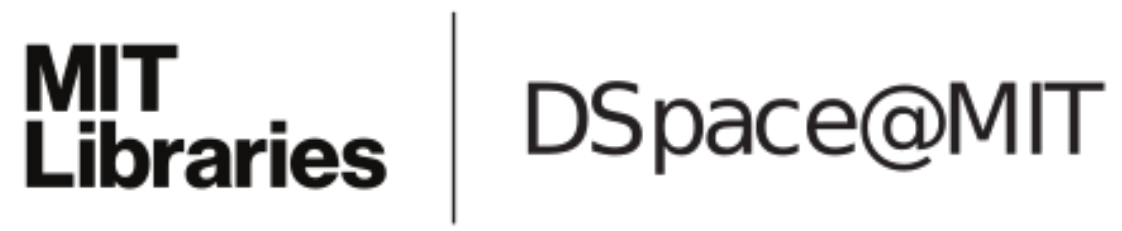

\author{
MIT Open Access Articles
}

Bayesian Model Selection Applied to the Analysis of Fluorescence Correlation Spectroscopy Data of Fluorescent Proteins in Vitro and in Vivo

The MIT Faculty has made this article openly available. Please share how this access benefits you. Your story matters.

Citation: Sun, Guangyu, Syuan-Ming Guo, Cathleen Teh, Vladimir Korzh, Mark Bathe, and Thorsten Wohland. "Bayesian Model Selection Applied to the Analysis of Fluorescence Correlation Spectroscopy Data of Fluorescent Proteins in Vitro and in Vivo." Anal. Chem. 87, no. 8 (April 21, 2015): 4326-4333.

As Published: http://dx.doi.org/10.1021/acs.analchem.5b00022

Publisher: American Chemical Society (ACS)

Persistent URL: http://hdl.handle.net/1721.1/99237

Version: Author's final manuscript: final author's manuscript post peer review, without publisher's formatting or copy editing

Terms of use: Creative Commons Attribution-Noncommercial-Share Alike 


\title{
Bayesian Model Selection Applied to the Analysis of Fluorescence Correlation Spectroscopy Data of Fluorescent Proteins in Vitro and in Vivo
}

\author{
Guangyu Sun ${ }^{\dagger,}$, Ming Guo ${ }^{\S}$, Cathleen Teh", Vladimir Korzh ${ }^{\|, \#, ~ M a r k ~ B a t h e ~}{ }^{\S}$, and Thorsten \\ Wohland $\dagger, \neq, \#,{ }^{*}$ \\ tDepartment of Chemistry, National University of Singapore, 117543 Singapore \\ ¥Centre for Bioimaging Sciences, National University of Singapore, 117557 Singapore \\ $\S$ Laboratory for Computational Biology and Biophysics, Department of Biological Engineering, \\ Massachusetts Institute of Technology, Cambridge, Massachusetts 02139, United States \\ "Institute of Molecular and Cell Biology, Agency for Science, Technology and Research, 138673 \\ Singapore \\ \#Department of Biological Sciences, National University of Singapore, 117543 Singapore
}

\begin{abstract}
Fluorescence correlation spectroscopy (FCS) is a powerful technique to investigate molecular dynamics with single molecule sensitivity. In particular, in the life sciences it has found widespread application using fluorescent proteins as molecularly specific labels. However, FCS data analysis and interpretation using fluorescent proteins remains challenging due to typically low signal-to-noise ratio of FCS data and correlated noise in autocorrelated data sets. As a result, naive fitting procedures that ignore these important issues typically provide similarly good fits for multiple competing models without clear distinction of which model is preferred given the signalto-noise ratio present in the data. Recently, we introduced a Bayesian model selection procedure to overcome this issue with FCS data analysis. The method accounts for the highly correlated noise that is present in FCS data sets and additionally penalizes model complexity to prevent over interpretation of FCS data. Here, we apply this procedure to evaluate FCS data from fluorescent proteins assayed in vitro and in vivo. Consistent with previous work, we demonstrate that model selection is strongly dependent on the signal-to-noise ratio of the measurement, namely, excitation intensity and measurement time, and is sensitive to saturation artifacts. Under fixed, low intensity excitation conditions, physical transport models can unambiguously be identified. However, at
\end{abstract}

\footnotetext{
(C) 2015 American Chemical Society

*Corresponding Author. Phone: (65) 6516-1248. Fax: (65) 6776-7882. twohland@nus.edu.sg. ASSOCIATED CONTENT

Supporting Information

Additional information as noted in text. This material is available free of charge via the Internet at http://pubs.acs.org.

Author Contributions

The study was conceived by V.K. and T.W.; experiments were performed by G.S. and C.T.; all authors contributed to data interpretation and manuscript preparation.

The authors declare no competing financial interest.
} 
excitation intensities that are considered moderate in many studies, unwanted artifacts are introduced that result in nonphysical models to be preferred. We also determined the appropriate fitting models of a GFP tagged secreted signaling protein, Wnt3, in live zebrafish embryos, which is necessary for the investigation of Wnt 3 expression and secretion in development. Bayes model selection therefore provides a robust procedure to determine appropriate transport and photophysical models for fluorescent proteins when appropriate models are provided, to help detect and eliminate experimental artifacts in solution, cells, and in living organisms.

\section{Abstract}

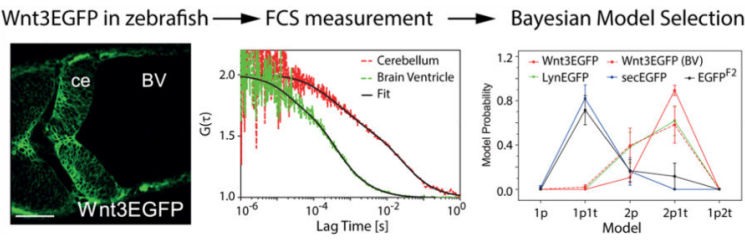

Fluorescence correlation spectroscopy (FCS) is a technique to measure molecular dynamics at the single molecule level. The use of fluorescent proteins has broadened its application to diverse basic questions in the life sciences. ${ }^{1-3}$ In FCS, physical properties of the process of interest are quantitatively inferred by fitting models to experimental data. Therefore, data interpretation relies on proper model selection. This is not a problem when dealing with known simple processes, such as diffusion of organic dyes in solution. However, the case becomes more complex for measurements in living systems for several reasons. First, the signal-to-noise ratio in live cell and in vivo measurements is usually not as good as in solution due to the increased background noise caused by autofluorescence and tissue scattering. In addition, the experimental conditions are restricted to low excitation intensity and short acquisition time due to photobleaching and optical saturation. ${ }^{4,5}$ Moreover, biological heterogeneity, including localization in different organelles and cellular chemical environments, cell movement, and different protein expression levels from cell to cell, renders the selection of the best model, which is defined according to Occam's razor or the principle of parsimony as the simplest model that describes the data, challenging. ${ }^{6,7}$ Objective, reproducible, and robust analysis of FCS data is becoming increasingly important due to the advent of high sensitivity camera-based detectors and advanced imaging modalities including single-plane illumination microscopy (SPIM) and total internal reflection fluorescence microscopy (TIRFM) that are now broadly accessible to diverse biological laboratories and researchers. ${ }^{8-11}$ While fluorescent proteins are by far the preferred labels in the life sciences, their photophysical properties further amplify this difficulty. Application of fluorescent proteins in FCS has a range of advantages, including ease of genetic labeling, controlled stoichiometry and, when working with transgenic cells and organisms, the ability to label intrinsically without the need to repeat labeling. However, fluorescent proteins also have a range of disadvantages. ${ }^{12-14}$ First, their brightness is typically lower compared to organic dyes, ${ }^{12}$ leading to lower signal-to-noise ratios. Second, they are less photostable, leading to faster bleaching and shorter measurement times. Finally, their photophysics are typically more complicated than that of organic dyes, ${ }^{15,16}$ rendering the selection of appropriate best-fitting models difficult. Thus, appropriate models 
describing the fluorescent protein autocorrelation function are essential for correct interpretation of biological FCS data.

It is not uncommon for a researcher to compare several models to determine the best representative one when using fluorescent proteins. ${ }^{17,18}$ For instance, it has been reported that both anomalous diffusion and two-species diffusion in two dimensions could be used to describe DiI-C12 diffusion in the plasma membrane. ${ }^{19}$ These results led to two different explanations for the underlying process. The same phenomenon was also observed in monitoring EGFP and EGFP-tagged proteins in nuclei. ${ }^{20}$ In the bacterium Escherichia coli, several diffusion models including an exchange model between a diffusing and an immobile state have been evaluated to characterize Min-protein dynamics. ${ }^{21}$ In Drosophila embryos, to determine the morphogen Bcd mobility in nuclei, different diffusion models including both simple and anomalous diffusion with different assumptions about EGFP photophysics were examined. ${ }^{22}$ However, only the simplest one species model was shown not to be able to adequately fit the data, the others giving equally good fits. Finally, the authors used the average value of diffusion times extracted from several possible models to estimate protein mobility. Comparing possible models one by one for each measurement is tedious and timeconsuming. More importantly, different models may lead to different interpretations of the underlying processes. Therefore, it is of great interest to have an objective and unbiased approach to FCS model evaluation.

FCS data analysis and interpretation is most commonly achieved using least-squares fitting of a set of possible predetermined models. Model selection is then based on reduced $\chi^{2}$ values obtained by each model. ${ }^{21,23}$ Improved model selection can be achieved by maximum likelihood estimation (MLE). ${ }^{24-26}$ However, this tends to overfit the data. ${ }^{27-29}$ The recently proposed Bayesian approach to FCS data analysis provides a novel way to analyze and interpret FCS data using objective model selection. ${ }^{30,31}$ Recently this was applied in data analysis of fluorescence based techniques, such as single-particle tracking, ${ }^{32,33}$ super-resolution imaging, ${ }^{34}$ single-molecule fluorescence resonance energy transfer (FRET), ${ }^{35}$ and imaging total internal reflection FCS (ITIR-FCS). ${ }^{11}$ In FCS data analysis, the Bayesian model selection procedure appropriately penalizes model complexity and prevents overfitting. Moreover, the highly correlated noise embedded in autocorrelation data is also estimated and taken into consideration during the model selection process. This approach has been demonstrated to be able to resolve the triplet state of Fluorescein at appropriate excitation intensity and two diffusing components in mixtures of Atto565 and Atto565-labeled streptavidin with distinct ratios. ${ }^{31}$

In this article, we focus on the first hurdle in model selection, namely, the determination of the appropriate models for fluorescence proteins in different in vitro and in vivo environments. We first used three organic dyes, Atto488, Fluorescein and Rhodamine 6G, to examine the performance of the Bayesian approach to model selection under diverse biologically relevant experimental conditions. We found that model selection was excitation intensity dependent. We then applied the same methodology to determine the appropriate models for widely used fluorescent proteins, namely, EGFP, EYFP, and mCherry in buffer solution and in the CHO-K1 (Chinese hamster ovary) cell cytoplasm and nucleus under different experimental conditions. We show that one-component diffusion is sufficient to 
describe the autocorrelation functions (ACFs) under low excitation intensity and onecomponent diffusion with a triplet state under moderate excitation intensity. Moreover, we also analyzed fitting models for a fluorescent protein labeled plasma membrane targeting sequence (PMT-EGFP) in live cells and found that the two-component diffusion (with a triplet under moderate excitation intensity) represents the best fitting model. Finally, we applied this approach to the model determination for EGFP labeled Wnt3, a signaling protein, measured in live zebrafish embryos. Comparison with membrane-bound or secreted control proteins demonstrated that Wnt3 is detected not only in the cytoplasm or on the cell membrane but also as extracellular secreted complexes.

\section{MATERIALS AND METHODS}

Details of the Materials and Methods are provided in the Supporting Information. Data analysis was performed as described here briefly.

The normalized autocorrelation function $(\mathrm{ACF})$ can be written as

$$
G(\tau)=\frac{\langle F(t) F(t+\tau)\rangle}{\langle F(t)\rangle^{2}}
$$

where $F(t)$ is the fluorescence intensity at time $t ;\langle\rangle$ denotes the time average, and $\tau$ is the lag time.

The ACF for a photon-count trace from photon arrival times (PAT) is calculated by the photon-count products:

$$
G\left(\tau_{k}\right)=G\left(k \Delta \tau_{i}\right)=\frac{\frac{1}{M} \sum_{m=1}^{M} \delta n_{m} \delta n_{m+k}}{\bar{n}_{0} \bar{n}_{k}}
$$

with

$$
\bar{n}_{j}=\frac{1}{M} \sum_{m=1}^{M} n_{m+j}
$$

where $\Delta \mathrm{\tau}_{i}$ is sampling time or channel width; $T_{\mathrm{aq}}$ is acquisition time; $n_{m}$ is the photon count at $m \Delta \tau_{i}, \delta n_{m}=\left(n_{m}-n_{0}\right)$ and $\delta n_{m+k}=\left(n_{m+k}-n_{k}\right) ; M=\left(T_{\mathrm{aq}} / \Delta \tau_{i}-k\right)$, is the number of possible products $\delta n_{m} \delta n_{m+k}$.

Noise and noise covariance matrices in ACFs are estimated from the intensity trace using the blocking procedure. ${ }^{31}$ For each ACF, the minimal averaging time, beyond which the transformed samples of the photon-count products are no longer correlated, is determined. Then, the transformed samples can be calculated and used for the sample covariance matrix calculation. Model probabilities and parameter estimates are calculated using the Bayesian inference procedure described previously (Figure 1). ${ }^{30,31}$ For each condition, at least 12 PAT traces were recorded and the data were presented as the average calculated model probabilities with standard error of the mean (SEM). 


\section{RESULTS AND DISCUSSION}

\section{Model Selection Is Excitation Intensity Sensitive}

We used organic dyes, Atto488, Fluorescein, and Rhodamine 6G, to validate the model selection approach under various experimental conditions. The data quality or the signal-tonoise depends both on the fluorophore brightness and on the acquisition time. ${ }^{36}$ The brightness ( $\eta$, Supporting Information sections S1 and S7) is measured in counts per particle per second (cps) and can be increased within a certain range by increasing the excitation intensity. Therefore, the effect of excitation intensity and acquisition time on model selection was examined.

The signal-to-noise ratio of the ACFs increased with excitation intensity as expected (Figure 2, Supporting Information Figure S1). With increasing excitation intensity, the brightness increased linearly at low excitation intensity (below $8 \mathrm{~kW} / \mathrm{cm}^{2}$ ) for organic dyes (Supporting Information Figure S2A). At higher intensities, the rate of increase slowed down as saturation levels were reached, where the detection volume is no longer the assumed 3D Gaussian intensity profile but the profile is distorted and flattened in the center. ${ }^{37,38}$ This results in a larger observation volume and thus an over-estimated number of particles, increased background, and decreased brightness, as well as a larger apparent diffusion time extracted from fitting (Supporting Information Figure S5A). The saturation level of a particular fluorophore depends on its photophysical properties, including its absorption cross-section, lifetime, and propensity to transit to long-lived transient states, e.g., triplets or conformational isomers. ${ }^{38-41}$ The effect of the triplet state build-up can be clearly seen in the case of Fluorescein, whose brightness first plateaus and then drops with increasing triplet state population (Figure 2A and Supporting Information Figure S2A).

We evaluated the following models, which all assumed 3-dimensional (3D) diffusion: onecomponent diffusion (1p, eq S1 in the Supporting Information), two-component diffusion (2p, eq S5 in the Supporting Information), one-component diffusion with a triplet state (1p1t, eq S7 in the Supporting Information), two-component diffusion with a triplet state (2p1t, eq S10 in the Supporting Information), and one-component diffusion with two triplet states (1p2t, eq S12 in the Supporting Information). Model probabilities and selection are shown in the Supporting Information Figure S3. Under low excitation intensity, below 4 $\mathrm{kW} / \mathrm{cm}^{2}$, the $1 \mathrm{p}$ model is preferred in all cases. The fluorophore has a low triplet fraction at this low excitation intensity and the signal-to-noise ratio is not sufficiently high to allow the distinction of a triplet state in the microsecond range of the ACF. As the excitation intensity increases, the model preference transitions from $1 \mathrm{p}$ to $1 \mathrm{p} 1 \mathrm{t}$. This trend is the same for all three tested dyes. However, from $15 \mathrm{~kW} / \mathrm{cm}^{2}$, the preferred model starts to show a competition between $1 \mathrm{p} 1 \mathrm{t}$ and $2 \mathrm{p}$ for Atto 488 and Rhodamine $6 \mathrm{G}$, whereas $1 \mathrm{p} 1 \mathrm{t}$ stays dominant for Fluorescein. However, the 2p model is unphysical as the "fast" moving component shows a diffusion time faster than the triplet state of the $1 \mathrm{p} 1 \mathrm{t}$ model and the "slow" moving component has a diffusion time longer than that in 1p1t (Supporting Information Figure S5A,B). In addition, the fraction of the fast diffusion coefficient develops parallel to the triplet fraction in the $1 \mathrm{p} 1 \mathrm{t}$ model and increases with excitation intensity, a direct indication that the $2 p$ model is incorrect (Supporting Information Figure 
S5C). The reason for this is that the underlying model is no longer valid under saturation conditions, even at moderate excitation intensities of $17 \mathrm{~kW} / \mathrm{cm}^{2}$ for Atto 488 and Rhodamine 6G. These model selection results are consistent with previous findings that models including $2 \mathrm{p}$ and one-component anomalous diffusion are better descriptions with improved residuals. ${ }^{37}$ However, in the case of Fluorescein, where the triplet fraction reaches more than $40 \%$ under low excitation intensity, the selection process is successful for almost the entire excitation range examined (Supporting Information Figures S3B and S5C). In this case the triplet state is sufficiently large and can be easily distinguished, while for Atto488 and Rhodamine $6 \mathrm{G}$ the much lower triplet fraction at the given signal-to-noise ratio cannot be clearly distinguished from the functional form of a diffusing component. In agreement with this hypothesis, at intensities above $42 \mathrm{~kW} / \mathrm{cm}^{2}$ and a triplet fraction of more than $25 \%$, the model preference of Atto488 returns to the 1p1t model. The same phenomenon was also observed for Rhodamine 6G (Supporting Information Figure S3C). The results are consistent with the reported Rhodamine $6 \mathrm{G}$ excitation saturation from $60 \mu \mathrm{W}$ in one-photon FCS. ${ }^{38}$

Next, we evaluated whether acquisition time will influence the fitting and model selection process. Exciting samples with higher intensity to improve the signal-to-noise ratio is not always possible, especially for fluorophores sensitive to saturation and photobleaching. In such cases, increasing the acquisition time will be the better option. On the other hand, short acquisition times may be necessary for highly dynamic systems. Hence, data with acquisition times of $10,20,40,80$, and $120 \mathrm{~s}$ under both low excitation intensity of 2 $\mathrm{kW} / \mathrm{cm}^{2}$ and high excitation intensity of $25 \mathrm{~kW} / \mathrm{cm}^{2}$ (where the competition between $1 \mathrm{p} 1 \mathrm{t}$ and $2 p$ occurs) were analyzed (Supporting Information section S2). Under low excitation intensity, increasing acquisition time barely changes the model selection (Supporting Information Figure S6, circles on solid line), but it improves data quality by increasing the signal-to-noise ratio and thus reduces errors of extracted parameters (Supporting Information Figure S9). Under high excitation intensity, which potentially saturates the sample, longer measurement times increase the signal-to-noise ratio but lead also to increased probabilities for the incorrect $2 \mathrm{p}$ model as expected (Supporting Information Figure S6, squares on dashed line).

\section{Fluorescent Proteins in Vitro}

We then applied this approach to fluorescent proteins in phosphate-buffered saline (PBS) buffer solution. Similar to organic dyes, the signal-to-noise ratio increases for ACFs as the excitation intensity increases (Figure 2B) and the brightness showed a linear increase up to an intensity of $4 \mathrm{~kW} / \mathrm{cm}^{2}$ (Supporting Information Figure S2B), where the order of fluorophores in descending brightness is EYFP, EGFP, and mCherry. The evaluated models were the same as for organic dyes. A similar preference is observed but with the $2 \mathrm{p}$ model preferred already at lower excitation intensities than for the organic dyes (Figure 3B). The $2 p$ selection was dominant at $4 \mathrm{~kW} / \mathrm{cm}^{2}$ for EYFP and $8 \mathrm{~kW} / \mathrm{cm}^{2}$ for EGFP indicating a lower threshold for saturation compared to the organic dyes. For mCherry, the situation is different and the $2 \mathrm{p}$ model is only selected from $25 \mathrm{~kW} / \mathrm{cm}^{2}$ upward. Similar to Fluorescein, the 1p1t dominance for a large range of the excitation intensity is due to its large fraction of dark state(s) (Supporting Information Figures S4C and S5F). A high fraction of the 
monomeric red fluorescent protein (mRFP1) has been shown to reside in dark states. ${ }^{42,43}$ Even though mCherry shows better photostability, maturation, and tolerance for tagging compared to mRFP1, ${ }^{12,44}$ it is still reported to reside with about $20-40 \%$ probability in a dark state in one photon $\mathrm{FCS}^{14}$ and with above $70 \%$ probability in a less bright state in two photon FCS. ${ }^{45}$ For all three fluorescent proteins, there exists a fast photophysical process whose relaxation time decreased with increasing excitation intensity and whose fraction remained constant (Supporting Information Figure S5E,F). Therefore, this process is likely to be photoinduced isomerization, in which the isomerization frequency is increased with photon flux. ${ }^{15,46}$ This blinking process due to isomerization is described by the same equation describing triplet state dynamics in the ACF. Furthermore, note that for the fluorescent proteins the apparent diffusion time decreases with increasing excitation intensity (Supporting Information Figure S5D). This is due to photobleaching as the destruction of the fluorophore reduces the time the molecule is detected and thus apparently reduces the residence time of the molecule in the observation volume. Similar to organic dyes, the parameter values of $2 \mathrm{p}$ are similar to the $1 \mathrm{p} 1 \mathrm{t}$ (Supporting Information Figure S5D-F), with the "fast moving" component corresponding to the isomerization relaxation time and its fraction to the isomerization fraction.

It has been reported that there are multiple blinking processes for EGFP and its variant EYFP. ${ }^{15,47,48}$ However, here 1p1t is sufficient to describe the ACFs, and $1 \mathrm{p} 2 \mathrm{t}$ was never preferred within the range of tested excitation intensities. One possible reason is that the excitation intensity is in the lower range compared to the reported study, in which the excitation intensity was beyond $25 \mathrm{~kW} / \mathrm{cm}^{2} .{ }^{15}$ Since the final goal of this study is to determine the appropriate fitting model for the application of fluorescent proteins in biological studies, the excitation intensity is kept below $25 \mathrm{~kW} / \mathrm{cm}^{2}$. Under the lowest tested excitation intensity, $1 \mathrm{p}$ is enough to describe the ACFs for all three fluorescent proteins. Different acquisition times for the fluorescent proteins yielded similar observation as for the organic dyes (Supporting Information Figure S7). The only exception was mCherry under $25 \mathrm{~kW} / \mathrm{cm}^{2}$ excitation. In this case, since the $2 \mathrm{p}$ model was never preferred, the longer acquisition time helped increase the signal-to-noise ratio and thus distinguished the triplet and picked the $1 \mathrm{p} 1 \mathrm{t}$ over the $1 \mathrm{p}$ model.

\section{Fluorescent Proteins in Vivo}

With the above knowledge, we applied the analysis to fluorescent proteins in $\mathrm{CHO}$ cells. Data analysis in cells is generally more difficult and ambiguous due to the heterogeneity of the environment, autofluorescence, and higher background. Furthermore, limitations on experimental conditions, such as low excitation intensity and short acquisition time, render data analysis more challenging. In cells, the brightness of fluorescent proteins is lower and their ACFs are therefore noisier than when measured in solution (Supporting Information Figures S1 and S2) because of increased background and autofluorescence. Some studies showed different mobility of EGFP in cytoplasm and nucleus, ${ }^{20}$ whereas other studies showed very close values at different cellular compartments. ${ }^{49}$ Therefore, we performed measurements both in the cytoplasm and the nucleus. However, we found that the chosen area had no influence on our model selection (data not shown). The extracted diffusion times of proteins in the cytoplasm and nucleus were similar (Supporting Information Table S9). 
Hence, only results from the cytoplasm measurements are shown (Supporting Information Figure S4C-F).

The evaluated models were the same as for measurements in solution. We found that under low excitation intensity the 1p model was sufficient to describe the ACFs of EGFP and mCherry and the 1p1t model was preferred for EYFP (Supporting Information Figure S4F). Similar to measurements in solution, increasing excitation intensity induces a preference shift to the $2 \mathrm{p}$ model. The $2 \mathrm{p}$ model in cells is still the same as in solution, that is, an imitation of the 1p1t (Supporting Information Figure S5G-I). Because of the environmental change, the triplet fraction of EYFP increased and thus changed its preference from the $2 p$ model to $1 \mathrm{p} 1 \mathrm{t}$. This is in agreement with the Fluorescein results, in which the 1p1t model remained dominant under high excitation intensity due to its high triplet fraction.

In cells, the acquisition time cannot be as long as in solution due to photobleaching and phototoxicity, particularly at high laser excitation intensities due to the finite and relatively small cellular volume. Therefore, data with acquisition times of 5, 10, and $20 \mathrm{~s}$ under both low and high excitation intensities were analyzed (Supporting Information Figure S8). The acquisition time barely changed the model selection for EYFP and mCherry at both excitation intensities. Unlike in solution, even $20 \mathrm{~s}$ changed the EGFP model to the implausible $2 \mathrm{p}$ model at $2 \mathrm{~kW} / \mathrm{cm}^{2}$. Thus, it appears that $8 \mathrm{~kW} / \mathrm{cm}^{2}$ is a too high intensity for fluorescent proteins in $\mathrm{CHO}$ cells, since the $2 \mathrm{p}$ model dominates even with the shortest acquisition time.

These experiments were performed at room temperature (r.t.), but many experiments with mammalian cells are performed at $37^{\circ} \mathrm{C}$. Hence, model selection was also examined at this temperature. We evaluated organic dyes and fluorescent proteins in solution as well as fluorescent proteins in $\mathrm{CHO}$ cells. Our results show that the experimental temperature at 37 ${ }^{\circ} \mathrm{C}$ did not influence the model selection (data not shown), although inferred parameters changed somewhat because diffusivity is temperature dependent. The anomalous diffusion model is basically a replacement for the $1 \mathrm{p} 1 \mathrm{t}$ model but cannot be distinguished from it and thus was not considered further (details in Supporting Information section S3).

\section{Model Selection for Membrane Measurements}

In addition to FCS measurements in the cytoplasm, measurements were also performed on diffusing protein species residing on the membrane. To achieve this goal, a plasmid encoding EGFP fused to a plasma membrane targeting (PMT) sequence was used ${ }^{50,51}$ and transfected in CHO cells (Figure 4A). Evaluated models were 2-dimensional (2D) 1p, 1p1t, 2p, 2p1t, 1p2t (eqs S4, S9, S6, S11, S13 in the Supporting Information). We expected 1p1t to be the preferred model at moderate excitation intensities for the membrane-bound protein, as in the solution measurements of EGFP. However, the preferred models were twocomponent models: $2 \mathrm{p}$ at low to moderate excitation intensities and $2 \mathrm{p} 1 \mathrm{t}$ at high excitation intensities. Comparison with one-component models including a triplet/isomerization process required the knowledge of their characteristic lifetimes. We therefore determined the isomerization relaxation time, $\tau_{\text {iso }}$, from cytoplasmic EGFP measurements with a value of $20 \mu \mathrm{s}$ at $4 \mathrm{~kW} / \mathrm{cm}^{2}$ to $8 \mathrm{~kW} / \mathrm{cm}^{2}$. After fixing this value for the PMT-EGFP measurements, the other models could be compared. However, more than $80 \%$ of ACFs cannot be well 
explained by the $1 \mathrm{p} 1 \mathrm{t}, 2 \mathrm{p} 1 \mathrm{t}$, and $1 \mathrm{p} 2 \mathrm{t}$ models, because model parameters (diffusion time, triplet time, and fraction) were unreasonable, with diffusion times exceeding $1 \mathrm{~s}$ and triplet times being smaller than $0.1 \mathrm{~ns}$.

Model probabilities are plotted in Figure 4A with model parameters shown in the Supporting Information section S4. For a single nonfunctional protein, one would expect a onecomponent model $(1 \mathrm{p}, 1 \mathrm{p} 1 \mathrm{t}, 1 \mathrm{p} 2 \mathrm{t})$ to be selected. However, results show a preference for the $2 \mathrm{p}$ model, with a transition to the $2 \mathrm{p} 1 \mathrm{t}$ model as the excitation intensity increases. The dominant component is the slow moving component with a diffusion time around $25 \mathrm{~ms}$, typical for a membrane protein, with a fraction that is approximately $60 \%$. The other component diffuses considerably faster with a diffusion time on the same order as free EGFP measured in the cytoplasm, which was 350-500 $\mu$ s (corresponding to 8 to $2 \mathrm{~kW} / \mathrm{cm}^{2}$, respectively). As the excitation intensity increases, the apparent diffusion times of the two components decrease due to photobleaching. This is in accordance with the observation of EGFP measured in the cytoplasm. To further test whether the fast component is a data analysis artifact, a series of measurements based on pinhole size was performed (Supporting Information Figure S10F). As the pinhole size increases, the observation volume increases. Therefore, it will take a longer time for a fluorophore to pass through the volume resulting in a longer diffusion time. Both the fast and slow moving components showed a pinholedependent change, consistent with a diffusive origin of both components. The fast component could be a result of movement toward the membrane due to transport from the endoplasmic reticulum (ER) to the membrane and in the opposite direction due to dynamic internalization or recycling of the membrane. ${ }^{52,53}$ When overexpressed, PMT-EGFP is accumulated in the cytoplasm (Supporting Information Figure S10B). FCS was then performed in the cytoplasm under $4 \mathrm{~kW} / \mathrm{cm}^{2}$ excitation (cross in Figure S10B in the Supporting Information). The data are normalized and plotted together with membrane measurements in Figure 4B, and the extracted fitting parameters are listed in Table S2 in the Supporting Information. The diffusion time of PMT-EGFP in the cytoplasm is close to that of free cytosolic EGFP, i.e., $480 \mu$ s at $4 \mathrm{~kW} / \mathrm{cm}^{2}$, and similar to the fast component of Wnt3EGFP detected in the membrane measurements (see next section). This supports the hypothesis that for membrane measurements the 2 p model is most suitable.

\section{Model Selection for Measurements in Zebrafish}

In view of the robustness of the Bayesian model selection approach, this methodology was employed to evaluate transport models for EGFP labeled proteins measured in live zebrafish embryos. Zebrafish Wnt3 is a lipid modified signaling protein and is essential for neural development and cell proliferation. ${ }^{54,55} \mathrm{Wnt} 3$-EGFP has been shown to be functional (unpublished work). FCS analysis of its transport in its native in vivo environment requires determination of the appropriate model.

The construction of the EGFP-labeled proteins is detailed in Supporting Information section S8. Briefly, because of the lipid modification of the membrane localization domain of Wnt3, Wnt3EGFP locates on the membrane and some of it is secreted. LynEGFP, used as a negative control, is a membrane-marker protein. Similar to PMT-EGFP, it is on the membrane due to the membrane localization domain of the Lyn protein. ${ }^{56} \mathrm{EGFP}^{\mathrm{F} 2}$, EGFP 
expressed under control of the Wnt3-promoter, is present in the cytoplasm and thus used to quantify the intracellular protein mobility. The extracellular protein mobility is represented by the secreted EGFP (secEGFP), which is EGFP tagged with a secretion domain. ${ }^{57}$ Their expression in the zebrafish brain is shown in Supporting Information Figure S11.

To describe the distribution of membrane located Wnt3EGFP and LynEGFP, the following fitting models were evaluated: 2D 1p, 1p1t, 2p, 2p1t, 1p2t. To describe secretion of Wnt3EGFP and secEGFP into the brain ventricle, which is a fluid-filled internal cavity, as well as that of intracellular $\mathrm{EGFP}^{\mathrm{F} 2}$, the following fitting models were evaluated: 3D 1p, $1 \mathrm{p} 1 \mathrm{t}, 2 \mathrm{p}, 2 \mathrm{p} 1 \mathrm{t}, 1 \mathrm{p} 2 \mathrm{t}$. The triplet/isomerization time was fixed at $20 \mu \mathrm{s}$ as for PMT-EGFP. The experimental conditions used were $15 \mu \mathrm{W}$ excitation power (corresponding to excitation intensity $6 \mathrm{~kW} / \mathrm{cm}^{2}$ ) with acquisition time $15 \mathrm{~s}$. The model probabilities are plotted in Figure 5. The best model for secEGFP and EGFP ${ }^{\mathrm{F} 2}$ is $1 \mathrm{p} 1 \mathrm{t}$; for Wnt3EGFP, LynEGFP, and PMTEGFP on the membrane it is $2 \mathrm{p} 1 \mathrm{t}$ model. The latter is influenced by freely diffusing Wnt3EGFP or LynEGFP located close to the membrane. However, for Wnt3EGFP secreted into the brain ventricle, $2 \mathrm{p} 1 \mathrm{t}$ is also preferred, albeit with very different diffusion times for the slow moving component. In this case, the mobility of the fast moving component is on the same order of the mobility of secEGFP. However, the diffusion time of the slow component is within a relatively large range from 1 to $50 \mathrm{~ms}$, which indicates a potentially wide distribution of complexes of Wnt3EGFP with other extracellular components ${ }^{57-59}$ in the brain ventricle.

\section{CONCLUSION}

We employed Bayesian model selection to perform objective multiple hypothesis testing of competing transport models for commonly used fluorescent proteins in vitro, in live cells, and in zebrafish embryos. Objective data analysis and interpretation in these contexts is crucial because autocorrelation functions of fluorescent protein are in general heterogeneous and complex, including multiple triplet, isomerization, and protonation/deprotonation states. ${ }^{16}$ Thus, knowledge of the best transport models for fluorescent proteins, consistent with the signal-to-noise level in experimental data is essential to avoid misinterpretation of FCS data sets from biological systems. Overall, we identified a range of parameters that crucially influence model selection. First, at low laser intensity, the preferred models for cytoplasmic fluorescent proteins consist of one-component diffusion with an isomerization component ( $1 \mathrm{p} 1 \mathrm{t})$ under all experimental conditions examined, despite the large range of possible photophysical processes for fluorescent proteins. This implies that given the signalto-noise ratio attainable at this laser intensity, no other photophysical processes can be detected, although this clearly does not imply that they do not exist. Second, with increasing laser intensity, the preferred model transitions to a two-component model (2p). This is not a reflection of the actual physical state of the fluorescent protein but a result of saturation, which is known to be a deficiency in our ability to model FCS data sets based on autocorrelation analysis, not a shortcoming of the model selection procedure itself. ${ }^{38}$ Saturation changes the underlying assumption in FCS of a simple Gaussian laser profile in the focal volume, which leads to deviations from the classical FCS diffusion model and therefore incorrect model selection. Thus, simply increasing laser intensity does not improve our ability to resolve underlying physical transport processes but instead introduces artifacts 
in our data interpretation. This issue is resolved if the isomerization or triplet state is sufficiently strong to be identified. In the case of mCherry, which possesses a large isomerization/triplet fraction, the $1 \mathrm{p} 1 \mathrm{t}$ model is valid for a wide intensity range, even when saturation is already reached.

When applied to membrane-bound proteins in cells and embryos, Bayesian model selection results consistently in two-component models with a single photophysical state (2p1t) with diffusion times indicating the presence of a membrane bound and a possible free cytosolic component. In addition, in the brain ventricle the preferred model for Wnt3 is also the 2p1t model, indicating that $\mathrm{Wnt} 3$ is found free but also in various complexes with other proteins.

This work demonstrates that Bayesian model selection provides a means to identify the simplest model consistent with FCS data. However, it also reminds us that model selection is always only performed on the set of models provided as the competing set of hypotheses to be tested. Thus, if important photophysical or other physical processes are present that are not described by any of these models, then the simplest model that best fits the data will still be selected, but interpretation of its meaning clearly needs to be performed with caution. In all cases, experimental controls are essential to test for the presence of possible artifacts such as saturation, which we do not currently have the ability to resolve using analytical autocorrelation function models. In summary, proper interpretation of FCS data in the context of the biological systems explored here is only possible when keeping experimental parameters within a relatively narrow range, avoiding saturation but with sufficiently high signal-to-noise ratio, and by using proper experimental controls to test model-based interpretations in all cases.

\section{Supplementary Material}

Refer to Web version on PubMed Central for supplementary material.

\section{ACKNOWLEDGMENTS}

T.W. gratefully acknowledges funding from the Ministry of Education of Singapore (Grants MOE2012-T2-1-101, R-154-000-543-112), and G.S. is a recipient of a National University of Singapore Graduate Scholarship. Funding from the NSF Physics of Living Systems (to S.-M.G. and M.B.) is gratefully acknowledged. Research reported in this publication was supported by the National Institute of Mental Health of the National Institutes of Health under Award Number U01MH106011 (to S.-M.G. and M.B.). V.K. and C.T. are supported by an Institute of Molecular and Cell Biology Institutional Grant from the Agency for Science, Technology, and Research (A*STAR) of Singapore. V.K. and C.T. are also supported by A*STAR Nutrition and Food Science grant (Grant 1121770041).

\section{REFERENCES}

1. Müze J, Ohrt T, Schwille P. Laser Photon. Rev. 2011; 5:52-67.

2. Ries J, Schwille P. Bioessays. 2012; 34:361-368. [PubMed: 22415816]

3. Macháň R, Wohland T. FEBS Lett. 2014; 588:3571-3584. [PubMed: 24726724]

4. Delon A, Usson Y, Derouard J, Biben T, Souchier C. Biophys. J. 2006; 90:2548-2562. [PubMed: 16428281]

5. Tcherniak A, Reznik C, Link S, Landes CF. Anal. Chem. 2009; 81:746-754. [PubMed: 19093758]

6. Milon S, Hovius R, Vogel H, Wohland T. Chem. Phys. 2003; 288:171-186.

7. Hac AE, Seeger HM, Fidorra M, Heimburg T. Biophys. J. 2005; 88:317-333. [PubMed: 15501937] 
8. Kannan B, Guo L, Sudhaharan T, Ahmed S, Maruyama I, Wohland T. Anal. Chem. 2007; 79:44634470. [PubMed: 17489557]

9. Wohland T, Shi X, Sankaran J, Stelzer EH. Opt. Express. 2010; 18:10627-10641. [PubMed: 20588915]

10. Singh AP, Krieger JW, Buchholz J, Charbon E, Langowski J, Wohland T. Opt. Express. 2013; 21:8652-8668. [PubMed: 23571955]

11. Guo SM, Bag N, Mishra A, Wohland T, Bathe M. Biophys. J. 2014; 106:190-200. [PubMed: 24411251]

12. Shaner NC, Steinbach PA, Tsien RY. Nat. Methods. 2005; 2:905-909. [PubMed: 16299475]

13. Chudakov DM, Matz MV, Lukyanov S, Lukyanov KA. Physiol. Rev. 2010; 90:1103-1163. [PubMed: 20664080]

14. Foo YH, Naredi-Rainer N, Lamb DC, Ahmed S, Wohland T. Biophys. J. 2012; 102:1174-1183. [PubMed: 22404940]

15. Widengren J, Mets U, Rigler R. Chem. Phys. 1999; 250:171-186.

16. Widengren J, Terry B, Rigler R. Chem. Phys. 1999; 249:259-271.

17. Brock R, Hink MA, Jovin TM. Biophys. J. 1998; 75:2547-2557. [PubMed: 9788950]

18. Gennerich A, Schild D. Biophys. J. 2000; 79:3294-3306. [PubMed: 11106632]

19. Schwille P, Korlach J, Webb WW. Cytometry. 1999; 36:176-182. [PubMed: 10404965]

20. Wachsmuth M, Waldeck W, Langowski J. J. Mol. Biol. 2000; 298:677-689. [PubMed: 10788329]

21. Meacci G, Ries J, Fischer-Friedrich E, Kahya N, Schwille P, Kruse K. Phys. Biol. 2006; 3:255263. [PubMed: 17200601]

22. Abu-Arish A, Porcher A, Czerwonka A, Dostatni N, Fradin C. Biophys. J. 2010; 99:L33-L35. [PubMed: 20712981]

23. Meseth U, Wohland T, Rigler R, Vogel H. Biophys. J. 1999; 76:1619-1631. [PubMed: 10049342]

24. Eggeling C, Berger S, Brand L, Fries JR, Schaffer J, Volkmer A, Seidel CAM. J. Biotechnol. 2001; 86:163-180. [PubMed: 11257530]

25. Enderlein J, Gregor I, Patra D, Fitter J. J. Fluoresc. 2005; 15:415-422. [PubMed: 15986160]

26. Sengupta P, Garai K, Balaji J, Periasamy N, Maiti S. Biophys. J. 2003; 84:1977-1984. [PubMed: 12609900]

27. Posada D, Buckley TR. Syst. Biol. 2004; 53:793-808. [PubMed: 15545256]

28. Sivia, DS.; Skilling, J. Data Analysis: A Bayesian Tutorial. 2nd ed.. Oxford, U.K.: Oxford University Press; 2006.

29. Gregory, PC. Bayesian Logical Data Analysis for the Physical Sciences: A Comparative Approach with Mathematica Support. New York: Cambridge University Press; 2005.

30. He J, Guo SM, Bathe M. Anal. Chem. 2012; 84:3871-3879. [PubMed: 22423978]

31. Guo SM, He J, Monnier N, Sun GY, Wohland T, Bathe M. Anal. Chem. 2012; 84:3880-3888. [PubMed: 22455375]

32. McHale K, Berglund AJ, Mabuchi H. Biophys. J. 2004; 86:3409-3422. [PubMed: 15189843]

33. Monnier N, Guo S-M, Mori M, He J, Lénárt P, Bathe M. Biophys. J. 2012; 103:616-626. [PubMed: 22947879]

34. Cox S, Rosten E, Monypenny J, Jovanovic-Talisman T, Burnette DT, Lippincott-Schwartz J, Jones GE, Heintzmann R. Nat. Methods. 2012; 9:195-200. [PubMed: 22138825]

35. Bronson JE, Fei JY, Hofman JM, Gonzalez RL, Wiggins CH. Biophys. J. 2009; 97:3196-3205. [PubMed: 20006957]

36. Koppel DE. Phys. Rev. A. 1974; 10:1938-1945.

37. Hess ST, Webb WW. Biophys. J. 2002; 83:2300-2317. [PubMed: 12324447]

38. Nagy A, Wu JR, Berland KM. J. Biomed. Opt. 2005; 10:044015.

39. Pawley JB, Barry R. Opt. Eng. 1996; 35:2765-2766.

40. Enderlein J, Gregor I, Patra D, Fitter J. Curr. Pharm. Biotechnol. 2004; 5:155-161. [PubMed: 15078149]

41. Cianci GC, Wu JR, Berland KM. Microsc. Res. Tech. 2004; 64:135-141. [PubMed: 15352084] 
42. Hillesheim LN, Chen Y, Muller JD. Biophys. J. 2006; 91:4273-4284. [PubMed: 16980358]

43. Hendrix J, Flors C, Dedecker P, Hofkens J, Engelborghs Y. Biophys. J. 2008; 94:4103-4113. [PubMed: 18234806]

44. Shaner NC, Campbell RE, Steinbach PA, Giepmans BNG, Palmer AE, Tsien RY. Nat. Biotechnol. 2004; 22:1567-1572. [PubMed: 15558047]

45. Wu B, Chen Y, Muller JD. Biophys. J. 2009; 96:2391-2404. [PubMed: 19289064]

46. Widengren J, Seidel CAM. Phys. Chem. Chem. Phys. 2000; 2:3435-3441.

47. Schwille P, Kummer S, Heikal AA, Moerner WE, Webb WW. Proc. Natl. Acad. Sci. U.S.A. 2000; 97:151-156. [PubMed: 10618386]

48. Heikal AA, Hess ST, Baird GS, Tsien RY, Webb WW. Proc. Natl. Acad. Sci. U.S.A. 2000; 97:11996-12001. [PubMed: 11050231]

49. Chen Y, Muller JD, Ruan QQ, Gratton E. Biophys. J. 2002; 82:133-144. [PubMed: 11751302]

50. Chapple JP, Hardcastle AJ, Grayson C, Willison KR, Cheetham ME. Invest. Ophthalmol. Vis. Sci. 2002; 43:2015-2020. [PubMed: 12037013]

51. Liu P, Sudhaharan T, Koh RML, Hwang LC, Ahmed S, Maruyama IN, Wohland T. Biophys. J. 2007; 93:684-698. [PubMed: 17468161]

52. Steinman RM, Mellman IS, Muller WA, Cohn ZA. J. Cell Biol. 1983; 96:1-27. [PubMed: 6298247]

53. Mellman I. Annu. Rev. Cell. Dev. Biol. 1996; 12:575-625. [PubMed: 8970738]

54. Clements WK, Ong KG, Traver D. Dev. Dyn. 2009; 238:1788-1795. [PubMed: 19452545]

55. Pei YX, Brun SN, Markant SL, Lento W, Gibson P, Taketo MM, Giovannini M, Gilbertson RJ, Wechsler-Reya RJ. Development. 2012; 139:1724-1733. [PubMed: 22461560]

56. Haas P, Gilmour D. Dev. Cell. 2006; 10:673-680. [PubMed: 16678780]

57. Yu SR, Burkhardt M, Nowak M, Ries J, Petrasek Z, Scholpp S, Schwille P, Brand M. Nature. 2009; 461:533-U100. [PubMed: 19741606]

58. Mulligan KA, Fuerer C, Ching W, Fish M, Willert K, Nusse R. Proc. Natl. Acad. Sci. U.S.A. 2012; 109:370-377. [PubMed: 22203956]

59. Gross JC, Chaudhary V, Bartscherer K, Boutros M. Nat. Cell Biol. 2012; 14:1036-1045. [PubMed: 22983114] 

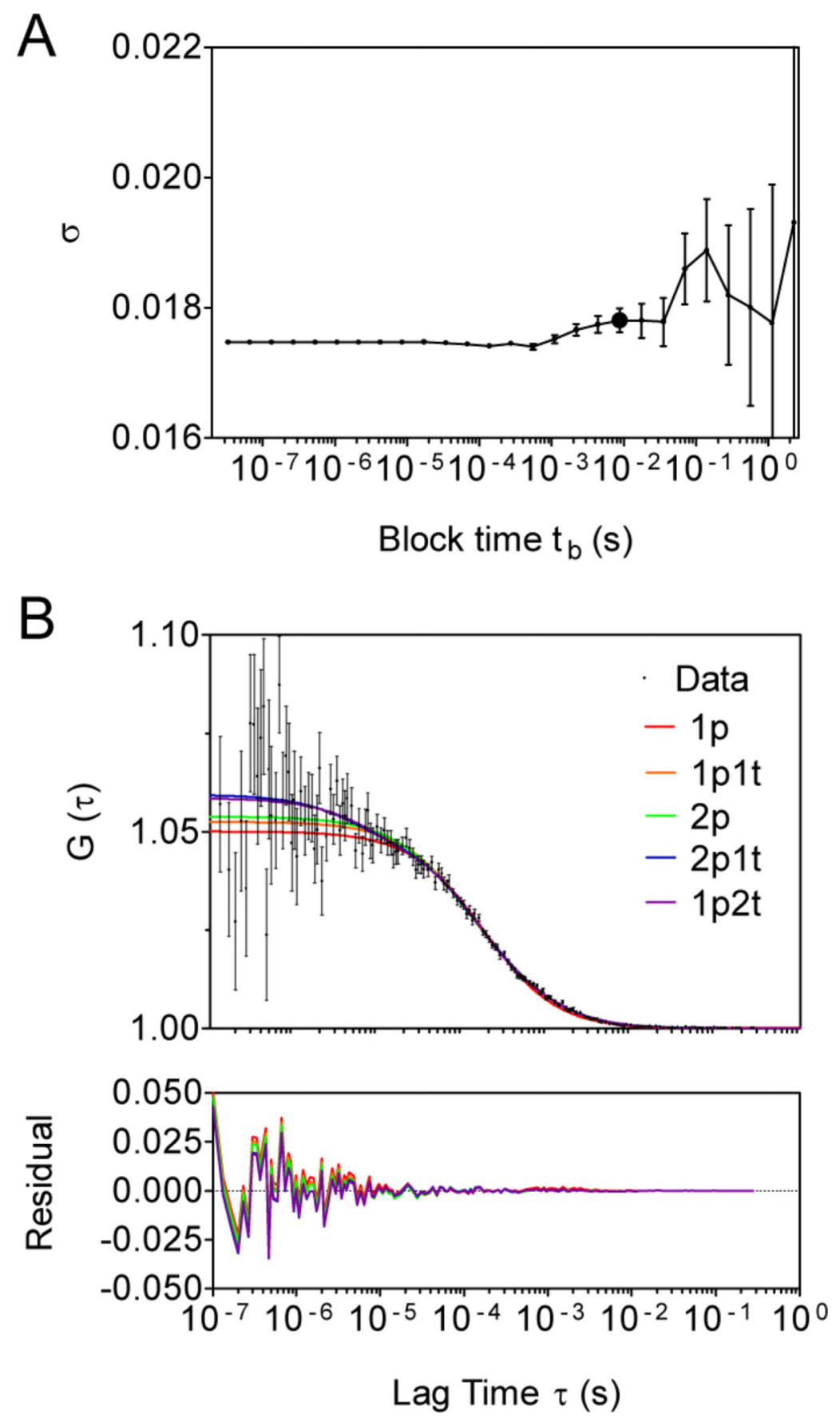

Figure 1.

Blocking transformation and fitting to tested models. (A) Estimated noise level as a function of block-time for $10 \mathrm{nM}$ EGFP at $10 \mathrm{~kW} / \mathrm{cm}^{2}$ with acquisition time of $40 \mathrm{~s}$. The fixed point is at approximately $8 \mathrm{~ms}$. Error bars: SD. (B) Fitting of evaluated models to ACF of EGFP calculated from the photon arrival time (PAT) trace. All fits were performed with 3D diffusion models. 


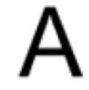

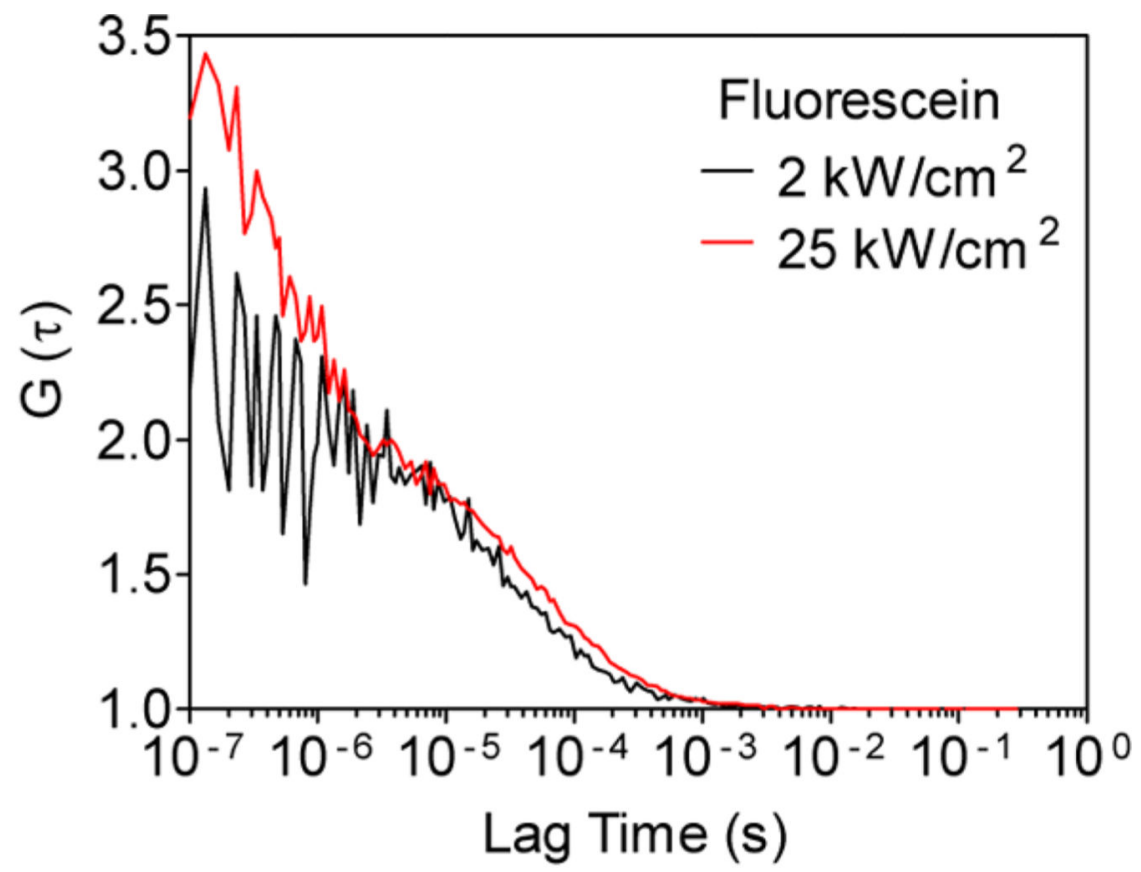

B

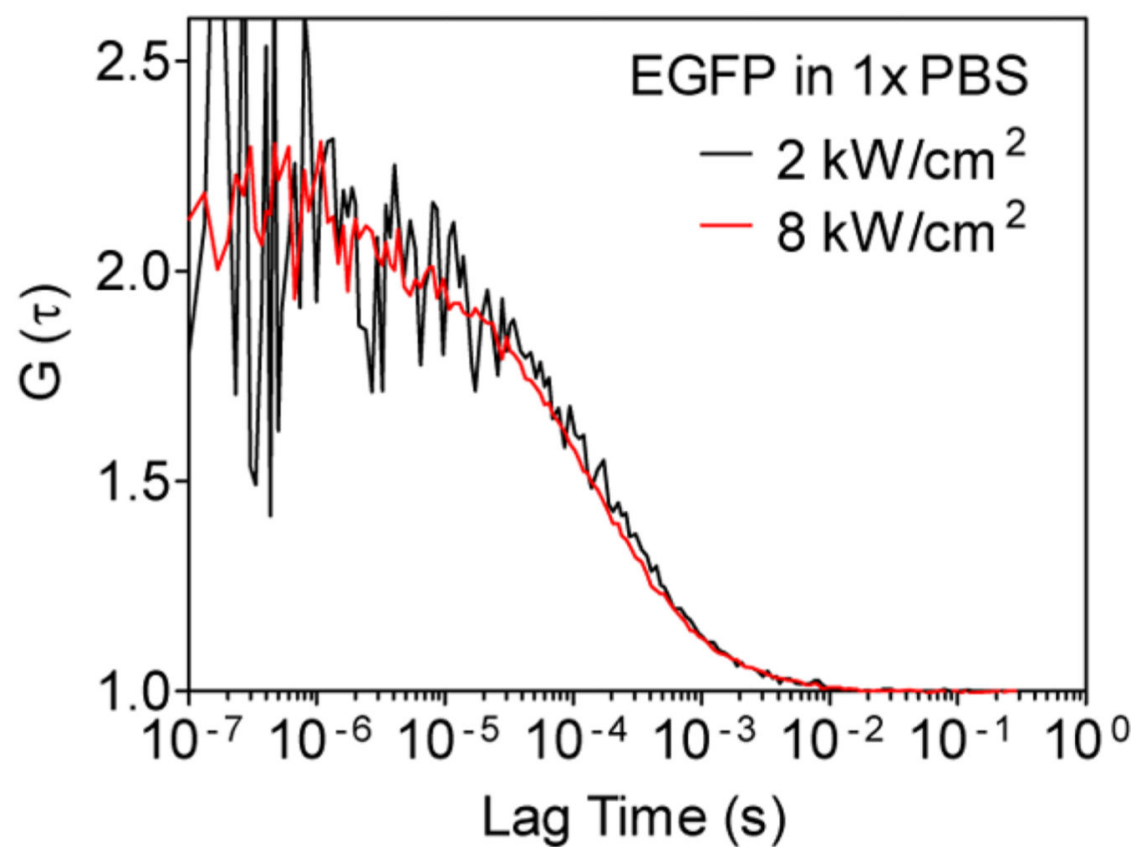

Figure 2.

ACFs under low and high excitation intensities of (A) Fluorescein and (B) EGFP in 1× PBS. 

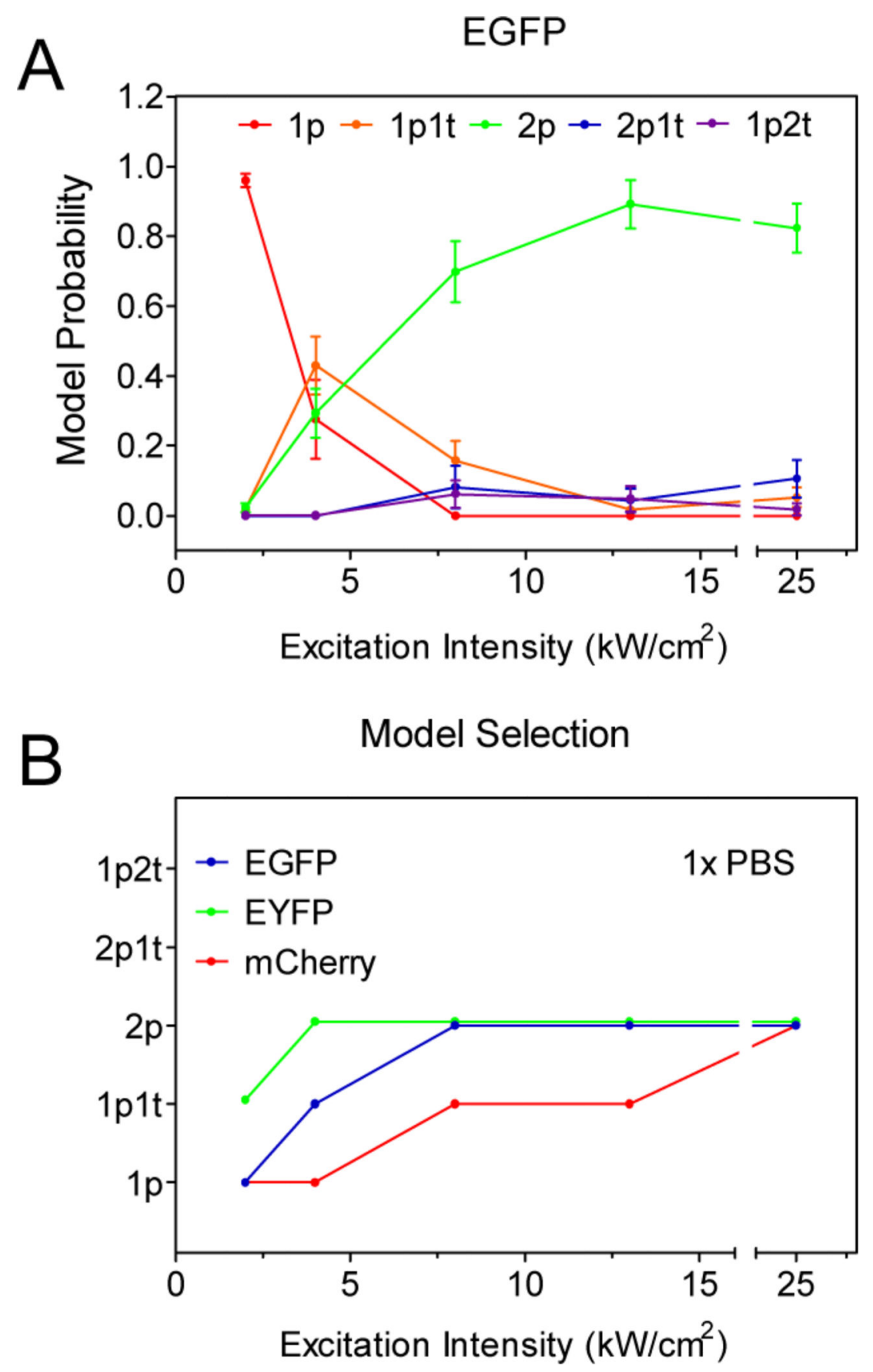

Figure 3.

Model probabilities and model selection of EGFP in $1 \times$ PBS at different excitation intensities. The acquisition time of these measurements was $40 \mathrm{~s}$. Model probabilities of (A) EGFP in $1 \times$ PBS. All fits were performed with 3D diffusion models. Model selection of (B) fluorescent proteins: EGFP (blue), EYFP (green), and mCherry (red) in 1× PBS. Error bar: SEM. 

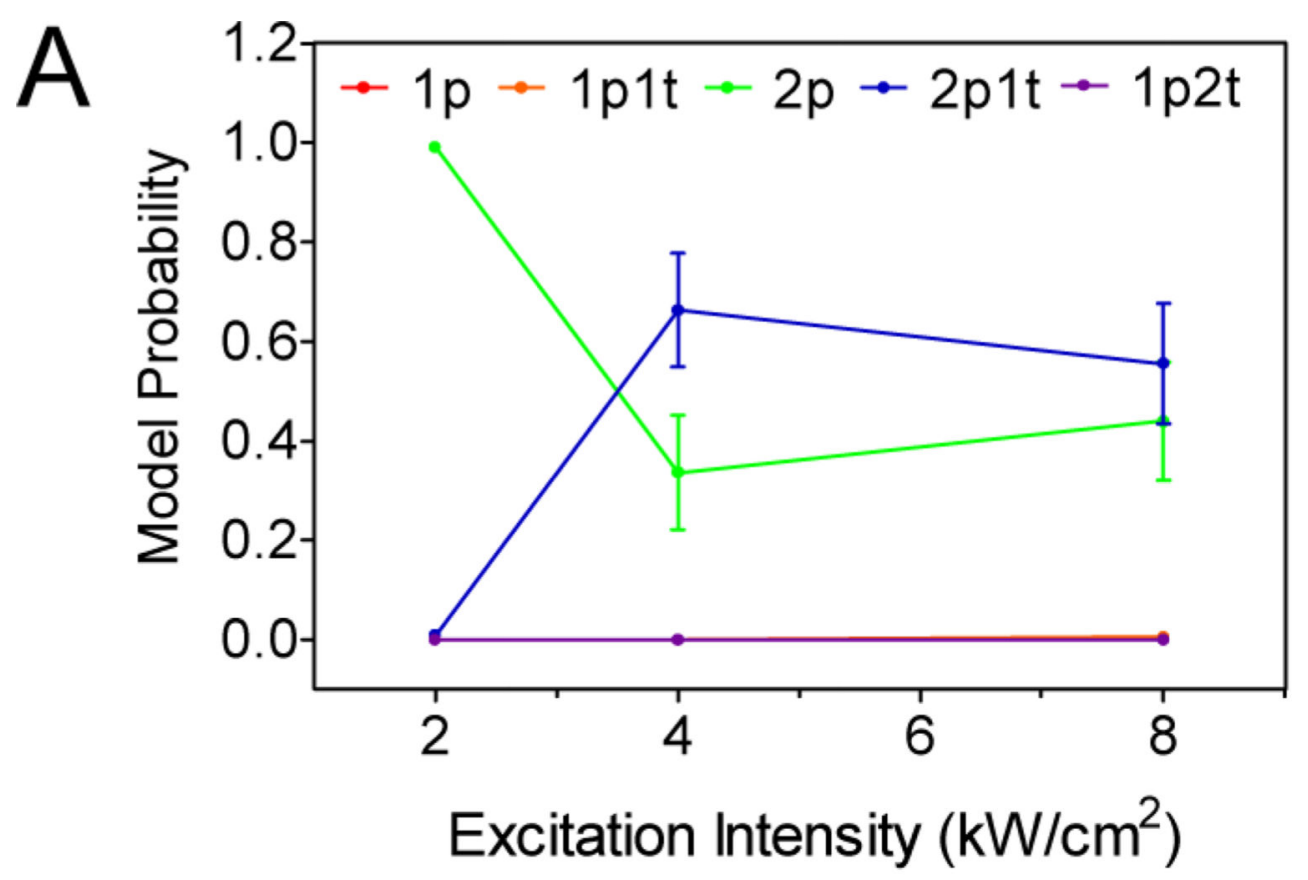

$D$

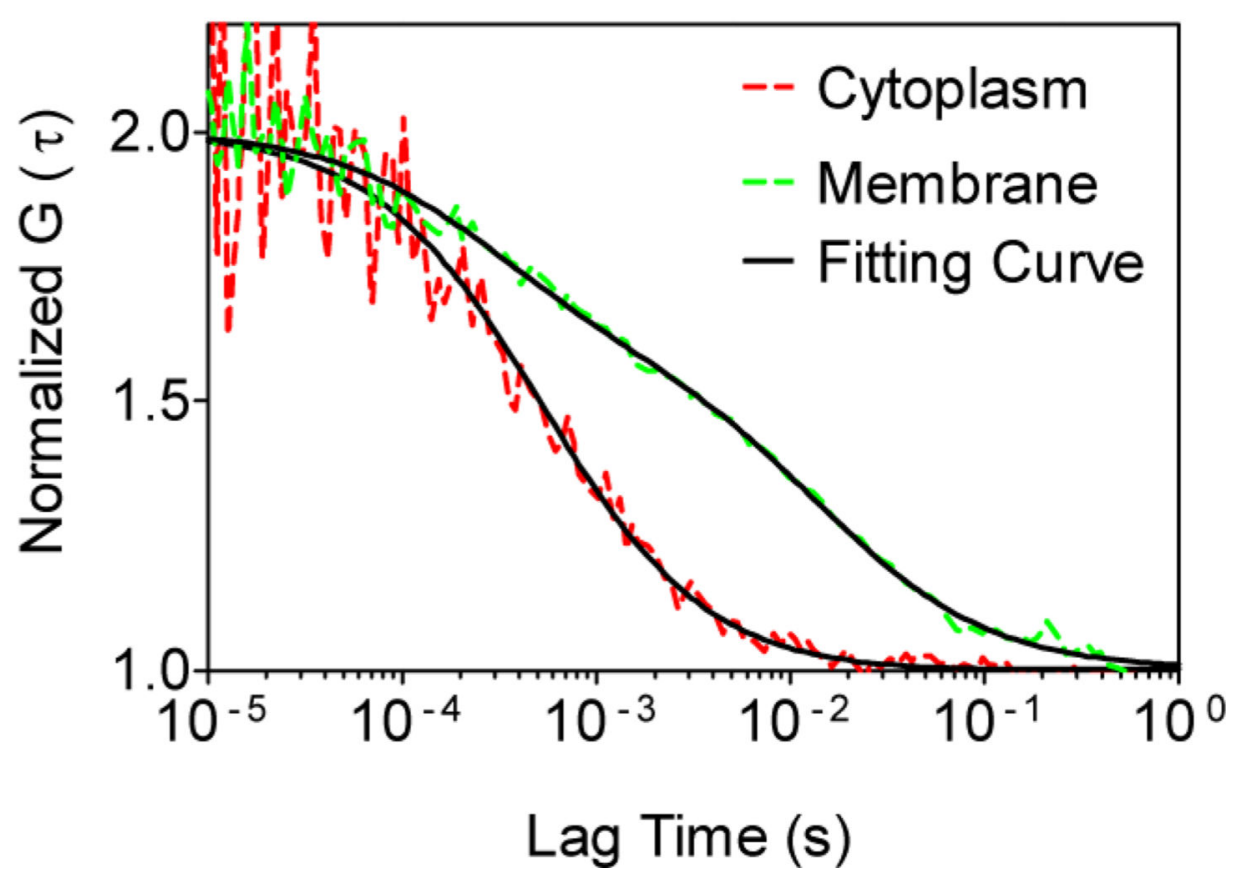

Figure 4.

Model probabilities PMT-EGFP in CHO cells and its ACFs on membrane and in the cytoplasm. (A) Model probabilities for PMT-EGFP on the membrane under different excitation intensities. All fits were performed with 2D diffusion models with the triplet blinking time fixed at $20 \mu \mathrm{s}$. Error bars: SEM. (B) Normalized ACFs of measurement in the cytoplasm (red dotted line) and on the membrane (green dotted line) with fits (black line). 


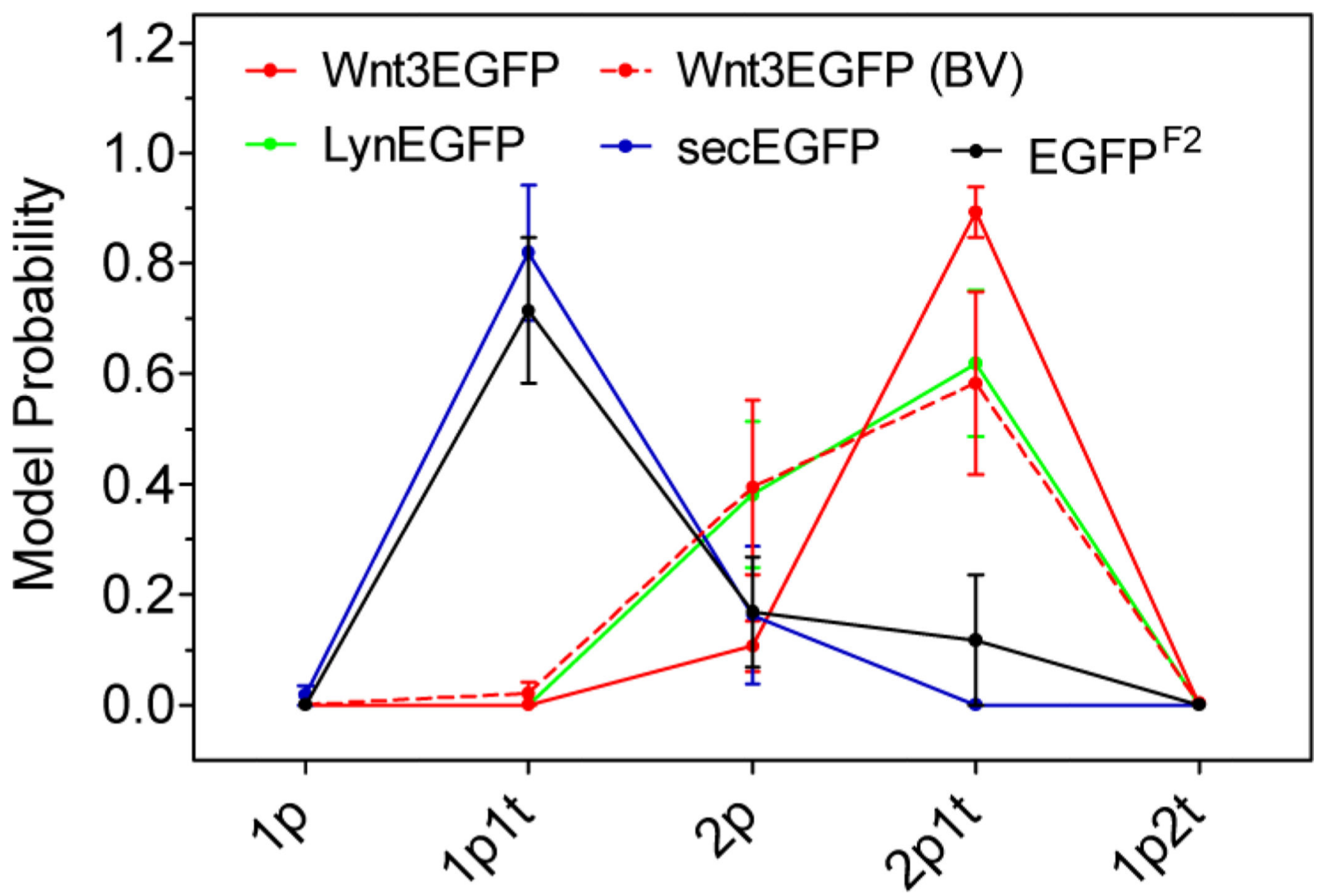

Figure 5.

Model probabilities of EGFP labeled proteins measured in zebrafish embryos. Error bars: SEM. BV: brain ventricle. 